\title{
HSP90 stabilizes B-cell receptor kinases in a multi-client interactome: PU-H71 induces CLL apoptosis in a cytoprotective microenvironment
}

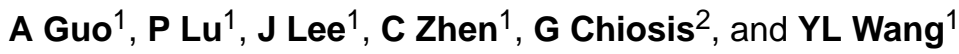 \\ ${ }^{1}$ Division of Genomic and Molecular Pathology, Department of Pathology, University of Chicago \\ ${ }^{2}$ Program in Chemical Biology, Memorial Sloan-Kettering Cancer Center, New York, NY, USA and \\ Department of Medicine, Memorial Sloan-Kettering Cancer Center, New York, NY, USA
}

\begin{abstract}
Chronic lymphocytic leukemia (CLL) is characterized by the accumulation of B cells in the hematopoietic system and lymphoid tissues. Although inhibitors targeting the B-cell receptor (BCR) pathway have been successful in the treatment of the disease, the underlying mechanisms leading to BCR over-activity in CLL are not fully understood. In this study, we found that HSP90, a highly conserved molecular chaperone, is overexpressed in CLL compared with resting B cells. HSP90 overexpression is accompanied by the overexpression of several BCR kinases including LYN, spleen tyrosine kinase, Bruton tyrosine kinase and AKT. Chemical and immuneprecipitation demonstrated that these BCR constituents are present in a multi-client chaperone complex with HSP90. Inhibition of HSP90 with PU-H71 destabilized the BCR kinases and caused apoptosis of CLL cells through the mitochondrial apoptotic pathway. Further, PU-H71 induced apoptosis in the presence of stromal co-culture or cytoprotective survival signals. Finally, genetic knockdown of HSP90 and its client AKT, but not BTK, reduced CLL viability. Overall, our study suggests that the chaperone function of HSP90 contributes to the over-activity of the BCR signaling in CLL and inhibition of HSP90 has the potential to achieve a multi-targeting effect. Thus, HSP90 inhibition may be explored to prevent or overcome drug resistance to single targeting agents.
\end{abstract}

\section{INTRODUCTION}

Chronic lymphocytic leukemia (CLL) is the most common adult leukemia in the Caucasian population. ${ }^{1}$ It is characterized by accumulation of mature lymphocytes in blood, bone marrow and lymphoid tissues. Recent studies have revealed that, in addition to having defective apoptosis, CLL is also proliferative, which relies on its tissue microenvironment for survival and proliferation. ${ }^{2}$ Intrinsically in the CLL tumor cells, B-cell receptor (BCR)

Correspondence: Professor YL Wang, Department of Pathology, University of Chicago, MC1089, N316, 5841 South Maryland Avenue, Chicago, IL 60637, USA. ylwang@bsd.uchicago.edu.

CONFLICT OF INTEREST

Memorial Sloan-Kettering Cancer Center holds the intellectual rights to PU-H71 (GC). Samus Therapeutics, of which GC has partial ownership, has licensed PU-H71. The remaining authors declare no conflict of interest.

Supplementary Information accompanies this paper on the Oncogene website (http://www.nature.com/onc) 
signaling is aberrantly active and represents one of the most important oncogenic pathways in CLL. ${ }^{3}$ The pathway can be therapeutically targeted with small molecule inhibitors of BCR kinases. Recent development of inhibitors to spleen tyrosine kinase (SYK), Bruton tyrosine kinase (BTK) and phosphoinositide $3^{\prime}$-kinase (PI3K), have generated remarkable responses in CLL. ${ }^{4-7}$ Ibrutinib (BTK inhibitor) and idelalisib (PI3K $\delta$ inhibitor), in particular, have been approved by Food and Drug Administration as either a single agent or in combination with other agents for the treatment of CLL. However, large-scale sequencing efforts failed to identify recurrent mutations in the BCR signaling components as mechanisms causing BCR activation in CLL, ${ }^{8-10}$ as opposed to ABC diffuse large B-cell lymphoma. The direct mechanisms leading to overactive BCR signaling in CLL remain elusive.

Heat shock protein 90 (HSP90) is an ATP-dependent molecular chaperone that is involved in cellular homeostasis. In malignancies, HSP90 is exploited by cancer cells in proper folding of many mutated, activated or overexpressed oncoproteins, including kinases and transcription factors. ${ }^{11,12}$ HSP90 inhibition has been explored in CLL. It has been shown that naturally occurring geldanamycin induces CLL apoptosis through destabilization of LYN, an early BCR kinase, and synthetic 17-DMAG or NVP-AUY922 induce CLL apoptosis by depletion of I $\kappa$ B kinase, an activator of nuclear factor- $\kappa \mathrm{B} .{ }^{13-15}$ However, there is lack of understanding of the expression and function of HSP90, the chaperone itself, in CLL. Herein, we show evidence that the HSP is overexpressed in CLL. It forms a complex with several BCR kinases including BTK and AKT that may account for BCR over-activity in CLL. We also show that PU-H71, an HSP90 inhibitor with a purine scaffold, induces BCR kinase depletion and induces apoptosis in the presence of microenvironmental survival stimuli. Finally, we demonstrate that small interfering RNA (siRNA) knockdown of HSP90 and its client, AKT, but not BTK, leads to CLL cell death.

\section{RESULTS}

\section{HSP90 protein and BCR kinases are overexpressed in CLL compared with normal cell counterparts}

To determine whether HSP90 is relevant in CLL, we first compared its levels of expression in primary CLL cells with normal peripheral blood mononuclear cells (PBMCs) or purified resting B cells using immunoblot analyses. Shown in Figure 1a, HSP90 was evidently upregulated in CLL cells $(N=10)$ compared with PBMC $(N=6)$ or resting B cells $(N=6)$ from healthy donors. We then investigated whether key kinases in the BCR pathway are coexpressed in CLL cells overexpressing HSP90. As shown in Figure 1a, SYK and BTK were highly abundant in a majority of CLL samples, whereas expression of LYN and AKT was more variable. However, normalized quantitative analysis revealed that all four proteins along with HSP90 are overexpressed in CLL compared with PBMC or resting B cells (Figure 1b). With normalization to glyceraldehyde 3-phosphate dehydrogenase (GAPDH), HSP90 protein expression in CLL was, on average, 5-fold of PBMC samples and 17-fold of resting B cells (Figure $1 \mathrm{~b} ; P<0.01$ ). 


\section{BCR kinases are present in the multi-client HSP90 complex}

We next investigated whether these BCR signaling proteins are components of HSP90 interactome. Immunoprecipitation was performed using anti-HSP90-conjugated beads in cell lysates from CLL. In addition, PU-H71-conjugated beads were also used in chemical precipitation reaction to pull down proteins associated with the drug-bound HSP90. Figure 2 showed that LYN, SYK, BTK and AKT were present in CLL cell lysates (lane As), in complex with PU-H71-bound HSP90 (lane Bs), as well as in complex with HSP90 (lane Cs), but not much in the precipitates pulled down with the control agarose beads (lane Ds). In contrast to these signaling proteins, the control protein GAPDH was detected only in CLL cell lysates, but not in either the PU-H71 or the HSP90 complex. These results show that the interactions of BCR signaling proteins with HSP90 are highly specific. Interestingly, BTK appears to be particularly enriched in the complex with HSP90 or PU-H71-bound HSP90 (Figure 2, lanes Bs and Cs). Taken together, these data show that several key BCR kinases are protected specifically by HSP90 in its multi-client interactome. The complex likely facilitates the interactions between BCR kinases and perhaps accounts, at least partially, for the aberrantly high BCR activity in CLL in the absence of BCR mutations.

\section{Expression of BCR kinases are maintained by the chaperon function of HSP90}

Thus far, we had demonstrated that levels of HSP90 are elevated and key kinases in BCR pathway are client proteins of HSP90 suggesting that BCR pathway kinases are chaperoned and maintained at high levels by HSP90. To demonstrate that stability of the BCR kinases depends on the function of HSP90, we tested whether HSP90 inhibition with PU-H71 would reduce their abundance. Immunoblotting was performed with cells treated with the HSP90 inhibitor. Figure 3 demonstrated that levels of LYN, SYK, BTK and AKT were all decreased by PU-H71 treatment in a dose and time-dependent manner (Figures 3a and b). Remarkably, even under the conditions of stromal co-culture with NKTert or HS-5 cells, PU-H71 treatment caused the decline and depletion of these BCR signaling kinases (Figure 3c). These effects are relatively specific as abundance of MEK and ERK were much less influenced by PU-H71 (Figures 3a-c).

\section{PU-H71 significantly reduces the viability of CLL cells but has minimal effects on PBMC or resting $B$ cells}

With its ability to decrease the abundance of BCR signaling components, we postulate that PU-H71 would have a strong anti-tumor effect in CLL. We treated CLL cells with increasing concentrations of PU-H71 and assessed cell viability using propidium iodide staining. Figure 4 a shows a time-dependent reduction of cell viability in 41 CLL patient samples. The cell death was significant in a range of concentrations from 0.25 to $10 \mu \mathrm{M}$ (Figure $4 \mathrm{~b}, N=$ 10). The lowest concentration used, $0.25 \mu \mathrm{M}$, is well below the clinical intra-tumor concentrations measured in patients, where concentrations as high as $0.5-8 \mu \mathrm{M}$ were recorded at $24 \mathrm{~h}$ post single-dose PU-H71. ${ }^{16}$ When the cases were analyzed according to established prognostic markers, the cellular response did not differ between different status of ZAP70, CD38, IGHV mutation or cytogenetic abnormalities (Supplementary Figure 1).

To demonstrate the selective cytotoxicity of PU-H71 on CLL cells, we also evaluated its effects on PBMC and resting B cells from healthy volunteers. No significant cell death was 
observed in both types of normal cells treated with $0.25-5 \mu \mathrm{M}$ of the compound (Figures $4 \mathrm{c}$ and d). Only at a clinically irrelevant high concentration of $10 \mu \mathrm{M}$, viability of resting B cells was reduced by PU-H71 treatment to $89 \pm 2.8 \%$ or by $\sim 11 \%$ with 72 -h drug exposure (Figure $4 \mathrm{~d}, P<0.01$ ). Taken together, these data suggest that higher HSP90 expression (Figure 1a) in CLL cells may be responsible for selective cytotoxicity of PU-H71 in CLL tumor cells. It is also possible that HSP90 is used differently in the tumor cellular context creating a vulnerability to HSP90 inhibitors as previously reported. ${ }^{17-20}$

\section{PU-H71 reduces CLL viability through the induction of mitochondrial apoptosis}

We next investigated the mechanism underlying PU-H71 induced CLL cell death. We examined cell apoptosis using Annexin V/7-Aminoactinomycin D double staining. Figure 5a shows that concentration- and time-dependent induction of apoptotic cell population (upper and lower right quadrants). Similar results were observed in all six CLL specimens tested (Supplementary Figure 2). To determine whether mitochondrial apoptotic pathway was engaged by PU-H71, we measured the degree of cytochrome C release in CLL cells after PU-H71 exposure. Figure 5b shows that mitochondria-associated cytochrome $\mathrm{C}$ was significantly reduced following PU-H71 treatment after $24 \mathrm{~h}$ suggesting significant amount of cytochrome $\mathrm{C}$ was released from mitochondria to cytoplasm (green vs red).

BCL-2 family proteins have a key role in the regulation of mitochondrial apoptosis in CLL. Anti-apoptotic proteins MCL-1, BCL-2 and XIAP are known to be overexpressed in CLL cells. ${ }^{21-24}$ We thus analyzed the effect of PU-H71 on these proteins. As shown in Figures $5 \mathrm{c}$ and d, MCL-1 was down-regulated by PU-H71 in a dose-dependent manner in CLL specimens $(N=6)$, and the reduction occurred at as early as $24 \mathrm{~h}$ post treatment. In contrast, BCL-2 and XIAP were downregulated in some cases but not others. Taken together, these data demonstrate that PU-H71 induces CLL cell apoptosis through the mitochondrial pathway, at least in part, via downregulation of MCL-1.

\section{PU-H71 antagonizes the survival signals from CLL microenvironment}

In vivo CLL cell survival and proliferation are heavily dependent on microenvironmental stimuli, including self-antigens, cell-cell contact with stromal cells and signaling through adhesion molecules, chemokines and cytokines, such as CD40L and IL-4. ${ }^{25,26} \mathrm{We}$ investigated whether HSP90 inhibition by PU-H71 can overcome the protective effects of the microenvironment.

Freshly isolated CLL cells were either cultured alone or co-cultured with stromal cell line NKTert with or without PU-H71 and cell viability was determined after $72 \mathrm{~h}$. Shown in Figure 6a, stromal cell co-culture resulted in a marked increase in cell viability $(N=14$, NKTert vs dimethylsulfoxide). Under this condition, cells maintained their sensitivity to PU$\mathrm{H} 71$ inhibition at a low dose of $0.5 \mu \mathrm{M}(\mathrm{NK}+\mathrm{PU}$ vs NKTert). We then evaluated the outcome of HSP90 inhibition on the pro-survival effect of soluble factors CD40 ligand/Interleukin-4 (CD40L/IL-4, Figure 6b). Similar to NKTert co-culture, CLL cells stimulated with CD40L/ IL-4 displayed higher viability (Stim vs dimethylsulfoxide), but $0.5 \mu \mathrm{M}$ PU-H71 effectively counteracted this pro-survival effect (Stim+PU vs Stim). Finally, PU-H71 also reduced tumor cell survival under the condition of immunoglobulin (Ig) stimulation although the 
baseline cell survival was minimally affected by the anti-Ig treatment (Supplementary Figure 3). Collectively, these studies demonstrate that inhibition of HSP90 can overcome the prosurvival signals delivered through either cell-cell contact or soluble factors provided by the tumor microenvironment.

\section{HSP90 knockdown impairs CLL tumor cell viability}

To demonstrate that the apoptosis induction by PU-H71 was caused by the specific inhibition of HSP90 molecule, we used RNA interference to specifically target HSP90. Primary CLL cells were transfected with a pool of siRNA against HSP90 or a pool of nontargeting siRNA. ${ }^{27}$ As shown in Figure 7a, the amount of HSP90 protein was reduced by siHSP with some case-to-case variability. HSP90 knockdown resulted in a significant reduction in cell viability comparing cells transfected with siHSP with those transfected non-targeting siRNAs (Figure 7b). These results indicate that HSP90 is essential for tumor cell survival and viability is compromised when the abundance of HSP90 is reduced. The results further suggest that the cellular effects of PU-H71 were mediated by its inhibition of HSP90.

\section{HSP90 client protein AKT, but not BTK, is essential for CLL viability}

To further prove that downregulation of the BCR pathway is required to kill CLL cells, direct physical reduction of BTK and AKT genes by siRNA in primary CLL cells was performed to see how cell survival is affected. As shown in Figure 8, siRNAs against BTK and $\mathrm{AKT}$ alone, or in combination ( $\mathrm{si} \mathrm{A}+\mathrm{B})$ effectively reduced the abundance of the intended target, BTK, AKT or both to approximately 30\%, in cells cultured alone (Figures $8 \mathrm{a}-\mathrm{c}$ ) or co-cultured with NKTert (Figures $8 \mathrm{~d}-\mathrm{f}$ ). The siRNA depletion was highly specific as siAKT had little effect on BTK and vice versa. Non-targeting control (NT) had little effect on reducing either molecule.

These siRNAs, along with the non-targeting control, were then transfected into six primary cell samples and cell viability was measured $48 \mathrm{~h}$ following the transfection. Figures $8 \mathrm{~g}$ and $\mathrm{h}$ show that the viability of CLL cells was significantly reduced by the AKT knockdown and by the AKT/BTK double knockdown $(\mathrm{siA}+\mathrm{B})$ with or without stromal co-culture. However, BTK knockdown alone did not significantly affect cell viability under either condition. This observation is consistent with our previous finding showing that BTK, as an early component of the BCR pathway, is not a major factor in CLL viability but is essential for CLL proliferation. ${ }^{28-30}$ These results support the notion that CLL killing by PU-H71 is mediated, at least in part, by HSP90 inhibition-induced down-regulation of AKT.

\section{DISCUSSION}

In this study, we aimed at defining the roles of HSP90 and exploring HSP90 inhibition in CLL, we demonstrated: (1) HSP90 is overexpressed in CLL that is accompanied by overexpression of several key components of the BCR signaling pathway including LYN, SYK, BTK and AKT; (2) HSP90 forms a chaperoning complex with the BCR kinases; (3) treatment of CLL with PU-H71 depletes the protein expression of the BCR kinases; (4) PUH71 induces CLL apoptosis through mitochondrial apoptosis pathway; (5) PU-H71 
antagonizes stroma-mediated pro-survival signals and causes apoptosis; and (6) knockdown of HSP90 and its client, AKT, but not BTK, significantly reduces CLL survival.

Notably, PU-H71 displayed little toxicity to normal resting B cells and PBMC in the concentration range from 0.25 to $5 \mu \mathrm{M}$. Given that intra-tumor concentrations of $1 \mu \mathrm{M}$ can be achieved in patients, concentrations tested in this study are clinically achievable and relevant. ${ }^{16}$ The low level of toxicity is consistent with the previous report that PU-H71 preferentially binds to the form of tumor-enriched HSP90, but not to the house-keeping form of HSP90. ${ }^{17}$

Our findings regarding PU-H71 are consistent with the previous reports by other groups showing that HSP90 inhibition delivers antitumor activity in CLL using other inhibitors including geldanamycin, its derivative 17-DMAG and chemically distinct novel agent NVPAUY922. ${ }^{13-15}$ In particular, Trentin et al. ${ }^{13}$ found that LYN is overexpressed in CLL with high constitutive activity and aberrant cytosolic localization. LYN is tightly bound to HSP90 and geldanamycin destabilizes LYN and reduces its kinase activity. In this study, we further characterized the HSP90 interactome and demonstrates that not only LYN, but also the enzymatic substrates of LYN, namely SYK and BTK, are present in the same complex with the chaperone.

Complex of HSP90/BCR components was recently identified in the ABC subtype of diffuse large B-cell lymphoma (DLBCL) using a comprehensive proteomic approach. ${ }^{20}$ Chronic active BCR signaling was known as a key oncogenic pathway in the ABC-DLBCL. ${ }^{31}$ Components of the BCR signaling including LYN, SYK and BTK are identified in the tumor-enriched HSP90 interactome and PU-H71 destabilizes their steady-state expression. ${ }^{20}$ Interestingly, in the case of CLL, we found that BTK is particularly enriched in the HSP90 complex relative to LYN and SYK, which is not the case in DLBCL (Figure 2). This may help explain the high sensitivity of CLL to the BTK inhibitors. However, this deduction will need to be further tested. Besides BTK, we found that AKT was also enriched in the HSP90 interactome, but AKT is not present in the chaperone complex in DLBCL. The finding is consistent with the notion that the functions of the chaperones are cell context dependent. ${ }^{11}$ AKT indeed has a critical role of in CLL viability as we further demonstrated by the siRNA knockdown experiment (Figure 7).

Unlike ABC-DLBCL, mutations in BCR pathway components are uncommon in CLL. However, drugs targeting the BCR pathway, such as ibrutinib and idelalisib, are highly successful generating therapeutic response in the majority of patients receiving the therapy. Previous studies suggest that extrinsic self-antigens or motifs in BCR molecules on the cell surface activate BCR signaling. ${ }^{32}$ Here, we provide evidence that intrinsically, overexpression of oncogenic HSP90 may be partially responsible for BCR over-activity by stabilizing BCR kinases and facilitating interactions of the kinase-substrate in the same multi-protein complex.

Finally, although targeting particular kinases of the BCR pathway is highly effective, resistance to ibrutinib has emerged. Along with others, we have shown that mutations in BTK and PLC $\gamma 2$ confer ibrutinib resistance by restoring the BCR pathway 
activity. ${ }^{28,30,33-35}$ We further demonstrated that resistant cells remain sensitive to other kinase inhibitors such as SYK and PI3K inhibitors. ${ }^{28}$ Thus, multi-targeting along the BCR pathway will likely generate a much stronger and lasting therapeutic effect. In this sense, PU-H71, with its ability to hit multiple BCR components, has a great potential to prevent and overcome resistance to single target agents.

\section{MATERIALS AND METHODS}

\section{Patients and healthy donor samples}

Peripheral blood samples from 41 B-CLL patients (Supplementary Table 1) were collected for this study. CLL diagnosis was based on the clinical and immunophenotypic criteria outlined by IWCLL criteria. Informed patient consents for the study were obtained according to the Declaration of Helsinki and these studies were approved by the Institutional Review Boards of the University of Chicago and Weill Cornell Medical College. The samples were obtained at least 3 months either before receiving treatment or untreated. Normal B or T cells were isolated from peripheral blood of healthy donors obtained from the New York Blood Center.

\section{Reagents and antibodies}

The following antibodies were used for immunoblotting assays: anti-AKT (\#9272), antiERK1/2 (\#4695), anti-GSK-3B (\#9315), anti-MCL-1 (\#4572), anti-SYK (\#2172) and antiLYN (\#4576) were purchased from Cell Signaling (Danvers, MA, USA). Anti-XIAP (\#610762) was purchased from BD Bioscience (San Jose, CA, USA). Anti-HSP90B (\#837159A) and anti-GAPDH (\#TAB1001) were purchased from Thermo Fisher Scientific (San Jose, CA, USA). Anti-BCL-2 (\#k2206) was purchased from Santa Cruz Biotechnology (Santa Cruz, CA, USA). Annexin V-fluorescein isothiocyanate and 7-

Aminoactinomycin $\mathrm{D}$ were obtained from BD Bioscience. The propidium iodide and InnoCyte Flow Cytometric Cytochrome $c$ Release kit were purchased from EMD Millipore (Kankakee, IL, USA). PU-H71 and PU-H71-conjugated agarose beads were obtained from Dr Gabriela Chiosis and were prepared as previously reported. ${ }^{17}$ Amaxa Solution V Nucleofector kit was purchased from Amaxa (Cologne, Germany). Pools of siRNA against human BTK, AKT and HSP90 were customarily designed and purchased from Thermo Scientific (Waltham, MA, USA).

\section{CLL and normal $B$ cells isolation}

CLL peripheral blood samples were processed as previously described..$^{28,29,36}$ Normal Bcell and CLL lymphocytes were isolated by negative selection using RosetteSep human Bcell enrichment cocktail (StemCell Technologies, Vancouver, BC, Canada), following the manufacturer's instructions. After separation, CLL cell purity $\left(\mathrm{CD} 19^{+} / \mathrm{CD}^{+}\right)$assessed by flow cytometry was $>95 \%$ in all cases. Cell viability was $\geq 90 \%$ determined by Trypan blue exclusion.

\section{Cell culture and co-culture conditions}

CLL cells were cultured in RPMI-1640 (Gibco, Grand Island, NY, USA) supplemented with $10 \%$ (v/v) fetal bovine serum at $37{ }^{\circ} \mathrm{C}$ with $5 \% \mathrm{CO}_{2}$ (Gibco). Stromal cell lines NKTert was 
cultured with RPMI-1640, and HS-5 with Dulbecco's modified Eagle's medium, supplemented with $20 \%$ fetal bovine serum, $50 \mathrm{U} / \mathrm{ml}$ penicillin, $50 \mu \mathrm{g} / \mathrm{ml}$ streptomycin and 2 $\mu \mathrm{M}$ glutamine (Gibco).

For co-culture experiments, stromal cells HS-5 or NKTert were seeded into 24-well plates (2 $\times 10^{4}$ cells $/ \mathrm{ml}$ per well) 1 day before the experiment. ${ }^{28-30}$ After confirming the confluence of stromal cell layer by phase contrast microscopy, CLL cells were added into the well at the ratio of 100:1 to stromal cells and were cultured for $72 \mathrm{~h}$. For ex vivo cytokine stimulation, CLL cells were treated with $100 \mathrm{ng} / \mathrm{ml} \mathrm{CD} 40 \mathrm{~L}$ and $10 \mathrm{ng} / \mathrm{ml} \mathrm{IL}-4$ for $72 \mathrm{~h}$. For ex vivo antigen stimulation, $a-\operatorname{IgM~} F\left(\mathrm{ab}^{\prime}\right)_{2}$ and $\mathrm{a}-\operatorname{IgD} \mathrm{F}\left(\mathrm{ab}^{\prime}\right)_{2}(10 \mu \mathrm{g} / \mathrm{ml}$ of each) were immobilized to tissue culture plates by overnight incubation at room temperature. Cell suspension was then added and cultured for $72 \mathrm{~h} .{ }^{37}$

\section{Analysis of cell viability and apoptosis}

CLL cells were treated with either vehicle (dimethylsulfoxide) or PU-H71 for 1-7 days at various concentrations in complete RPMI-1640 with or without stromal cells co-culture. Cell viability was measured by propidium iodide staining and flow cytometry analysis. $28-30,36,38,39$ Apoptosis was analyzed by double staining of 7-Aminoactinomycin D and fluorescein isothiocyanate-conjugated Annexin V (BD Biosciences). Flow cytometry was conducted using LSR2 flow cytometer (BD Biosciences) and the data were analyzed using FlowJo software (Flowjo LLC, Ashland, OR, USA).

\section{Measurement of mitochondrial cytochrome $c$ release}

The InnoCyte Flow Cytometric Cytochrome $c$ Release kit was used to quantify mitochondria-associated cytochrome c. ${ }^{36}$ Cells $\left(1 \times 10^{6}\right)$ were permeabilized and stained with anti-cytochrome $c$, according to the manufacturer's protocol. Ten thousand gated events were collected and analyzed by LSR2 flow cytometer and the data were analyzed using FlowJo software.

\section{Immunoblot analysis}

Immunoblot assays were conducted as described previously. ${ }^{28-30,36,40}$ Briefly, CLL cells were lysed in RIPA buffer with phosphotase and protease inhibitors (Calbiochem, San Diego, CA, USA). Concentration of lysate protein was determined by Protein Assay Reagent (Bio-Rad, Hercules, CA, USA). In all, $20 \mu \mathrm{g}$ proteins were separated by sodium dodecyl sulfate-polyacrylamide gel electrophoresis gel (Thermo Fisher Scientific) and electrotransfered onto polyvinylidene difluoride membranes (EMD Millipore). The membrane was probed with specific antibodies and the bands were detected with chemiluminescence detection kit (Thermo Fisher Scientific). The results of western blots were quantified by NIH ImageJ (NIH, Bethesda, MD, USA) and levels of protein expression were normalized to GAPDH (100\%).

\section{PU-H71 chemical precipitation and immunoprecipitation}

The CLL cell lysates (described above) were incubated with either PU-H71 beads, antiHSP90 beads or control beads for $4 \mathrm{~h}$ at room temperature. ${ }^{17}$ Beads were then washed three times with cold phosphate-buffered saline. Laemmli buffer was added and was boiled for 12 
min. After centrifugation, cells were loaded onto 4-12\% Bis-Tris gels. Equal amounts of cell lysates were loaded as a control. After transfer, polyvinylidene difluoride membranes were blocked in tris-buffered saline with tween 20 with $5 \%$ milk and incubated with appropriate dilutions of primary and secondary antibodies.

SiRNA nucleofection

siRNA against human HSP90, BTK or AKT ( $2 \mu \mathrm{g})$ were transfected into CLL cells using Amaxa Nucleofector II device (Amaxa, Cologne, Germany) and Solution V (Amaxa).

Nucleofector program U-15 was applied according to the manufacturer's instructions. ${ }^{28,29,40,41}$

\section{Statistical analysis}

Student's paired $t$-test was used for analyzing the statistical significance between two sample groups and one-way analysis of variance was used for multi-group comparison. All statistical analyses were conducted using Graphpad Prism (GraphPad, La Jolla, CA, USA). $P$-values of $<0.05$ were considered statistically significant.

\section{Supplementary Material}

Refer to Web version on PubMed Central for supplementary material.

\section{References}

1. Morton LM, Wang SS, Devesa SS, Hartge P, Weisenburger DD, Linet MS. Lymphoma incidence patterns by WHO subtype in the United States, 1992-2001. Blood. 2006; 107:265-276. [PubMed: 16150940]

2. Chiorazzi N, Rai KR, Ferrarini M. Chronic lymphocytic leukemia. N Engl J Med. 2005; 352:804815. [PubMed: 15728813]

3. Rai KR, Jain P. Chronic lymphocytic leukemia (CLL)-then and now. Am J Hematol. 2016; 91:330340. [PubMed: 26690614]

4. Byrd JC, Furman RR, Coutre SE, Flinn IW, Burger JA, Blum KA, et al. Targeting BTK with ibrutinib in relapsed chronic lymphocytic leukemia. N Engl J Med. 2013; 369:32-42. [PubMed: 23782158]

5. Furman RR, Sharman JP, Coutre SE, Cheson BD, Pagel JM, Hillmen P, et al. Idelalisib and rituximab in relapsed chronic lymphocytic leukemia. N Engl J Med. 2014; 370:997-1007. [PubMed: 24450857]

6. Advani RH, Buggy JJ, Sharman JP, Smith SM, Boyd TE, Grant B, et al. Bruton tyrosine kinase inhibitor ibrutinib (PCI-32765) has significant activity in patients with relapsed/refractory B-cell malignancies. J Clin Oncol. 2013; 31:88-94. [PubMed: 23045577]

7. Sharman J, Hawkins M, Kolibaba K, Boxer M, Klein L, Wu M, et al. An open-label phase 2 trial of entospletinib (GS-9973), a selective spleen tyrosine kinase inhibitor, in chronic lymphocytic leukemia. Blood. 2015; 125:2336-2343. [PubMed: 25696919]

8. Landau DA, Carter SL, Stojanov P, McKenna A, Stevenson K, Lawrence MS, et al. Evolution and impact of subclonal mutations in chronic lymphocytic leukemia. Cell. 2013; 152:714-726. [PubMed: 23415222]

9. Landau DA, Tausch E, Taylor-Weiner AN, Stewart C, Reiter JG, Bahlo J, et al. Mutations driving CLL and their evolution in progression and relapse. Nature. 2015; 526:525-530. [PubMed: 26466571] 
10. Puente XS, Bea S, Valdes-Mas R, Villamor N, Gutierrez-Abril J, Martin-Subero JI, et al. Noncoding recurrent mutations in chronic lymphocytic leukaemia. Nature. 2015; 526:519-524. [PubMed: 26200345]

11. Shrestha L, Patel HJ, Chiosis G. Chemical tools to investigate mechanisms associated with HSP90 and HSP70 in disease. Cell Chem Biol. 2016; 23:158-172. [PubMed: 26933742]

12. Scaltriti M, Dawood S, Cortes J. Molecular pathways: targeting hsp90-who benefits and who does not. Clin Cancer Res. 2012; 18:4508-4513. [PubMed: 22718860]

13. Trentin L, Frasson M, Donella-Deana A, Frezzato F, Pagano MA, Tibaldi E, et al. Geldanamycininduced Lyn dissociation from aberrant Hsp90-stabilized cytosolic complex is an early event in apoptotic mechanisms in B-chronic lymphocytic leukemia. Blood. 2008; 112:4665-4674. [PubMed: 18768392]

14. Hertlein E, Wagner AJ, Jones J, Lin TS, Maddocks KJ, Towns WH 3rd, et al. 17-DMAG targets the nuclear factor-kappaB family of proteins to induce apoptosis in chronic lymphocytic leukemia: clinical implications of HSP90 inhibition. Blood. 2010; 116:45-53. [PubMed: 20351313]

15. Walsby E, Pearce L, Burnett AK, Fegan C, Pepper C. The Hsp90 inhibitor NVP-AUY922-AG inhibits NF-kappaB signaling, overcomes microenvironmental cytoprotection and is highly synergistic with fludarabine in primary CLL cells. Oncotarget. 2012; 3:525-534. [PubMed: 22619113]

16. Gerecitano J, Modi S, Rampal R, Drilon AE, Fury MG, Gounder MM, et al. Phase I trial of the HSP-90 inhibitor PU-H71. J Clin Oncol. 2015; 33 abstr 2537.

17. Moulick K, Ahn JH, Zong H, Rodina A, Cerchietti L, Gomes DaGama EM, et al. Affinity-based proteomics reveal cancer-specific networks coordinated by Hsp90. Nat Chem Biol. 2011; 7:818826. [PubMed: 21946277]

18. Zong H, Gozman A, Caldas-Lopes E, Taldone T, Sturgill E, Brennan S, et al. A hyperactive signalosome in acute myeloid leukemia drives addiction to a tumor-specific Hsp90 species. Cell Rep. 2015; 13:2159-2173. [PubMed: 26628369]

19. Taldone T, Ochiana SO, Patel PD, Chiosis G. Selective targeting of the stress chaperome as a therapeutic strategy. Trends Pharmacol Sci. 2014; 35:592-603. [PubMed: 25262919]

20. Goldstein RL, Yang SN, Taldone T, Chang B, Gerecitano J, Elenitoba-Johnson K, et al. Pharmacoproteomics identifies combinatorial therapy targets for diffuse large B cell lymphoma. J Clin Invest. 2015; 125:4559-4571. [PubMed: 26529251]

21. Robertson LE, Plunkett W, McConnell K, Keating MJ, McDonnell TJ. Bcl-2 expression in chronic lymphocytic leukemia and its correlation with the induction of apoptosis and clinical outcome. Leukemia. 1996; 10:456-459. [PubMed: 8642861]

22. Balakrishnan K, Burger JA, Wierda WG, Gandhi V. AT-101 induces apoptosis in CLL B cells and overcomes stromal cell-mediated Mcl-1 induction and drug resistance. Blood. 2009; 113:149-153. [PubMed: 18836097]

23. Kitada S, Andersen J, Akar S, Zapata JM, Takayama S, Krajewski S, et al. Expression of apoptosis-regulating proteins in chronic lymphocytic leukemia: correlations with in vitro and in vivo chemoresponses. Blood. 1998; 91:3379-3389. [PubMed: 9558396]

24. Grzybowska-Izydorczyk O, Cebula B, Robak T, Smolewski P. Expression and prognostic significance of the inhibitor of apoptosis protein (IAP) family and its antagonists in chronic lymphocytic leukaemia. Eur J Cancer. 2010; 46:800-810. [PubMed: 20045309]

25. Hayden RE, Pratt G, Roberts C, Drayson MT, Bunce CM. Treatment of chronic lymphocytic leukemia requires targeting of the protective lymph node environment with novel therapeutic approaches. Leuk Lymphoma. 2012; 53:537-549. [PubMed: 21812539]

26. Burger JA. Nurture versus nature: the microenvironment in chronic lymphocytic leukemia. Hematol Am Soc Hematol Educ Program. 2011; 2011:96-103.

27. Andersen OS. Membrane proteins: through thick and thin. Nat Chem Biol. 2013; 9:667-668. [PubMed: 24141219]

28. Cheng S, Guo A, Lu P, Ma J, Coleman M, Wang YL. Functional characterization of BTK mutation that confers ibrutinib resistance: exploration of alternative kinase inhibitors. Leukemia. 2015; 29:895-900. [PubMed: 25189416] 
29. Guo A, Lu P, Galanina N, Nabhan C, Smith SM, Coleman M, et al. Heightened BTK-dependent cell proliferation in unmutated chronic lymphocytic leukemia confers increased sensitivity to ibrutinib. Oncotarget. 2015; 7:4598-4610.

30. Cheng S, Ma J, Guo A, Lu P, Leonard JP, Coleman M, et al. BTK inhibition targets in vivo CLL proliferation through its effects on B-cell receptor signaling activity. Leukemia. 2014; 28:649-657. [PubMed: 24270740]

31. Davis RE, Ngo VN, Lenz G, Tolar P, Young RM, Romesser PB, et al. Chronic active B-cellreceptor signalling in diffuse large B-cell lymphoma. Nature. 2010; 463:88-92. [PubMed: 20054396]

32. Duhren-von Minden M, Ubelhart R, Schneider D, Wossning T, Bach MP, Buchner M, et al. Chronic lymphocytic leukaemia is driven by antigen-independent cell-autonomous signalling. Nature. 2012; 489:309-312. [PubMed: 22885698]

33. Furman RR, Cheng S, Lu P, Setty M, Perez AR, Guo A, et al. Ibrutinib resistance in chronic lymphocytic leukemia. N Engl J Med. 2014; 370:2352-2354. [PubMed: 24869597]

34. Woyach JA, Furman RR, Liu TM, Ozer HG, Zapatka M, Ruppert AS, et al. Resistance mechanisms for the Bruton's tyrosine kinase inhibitor ibrutinib. N Engl J Med. 2014; 370:2286-2294. [PubMed: 24869598]

35. Liu TM, Woyach JA, Zhong Y, Lozanski A, Lozanski G, Dong S, et al. Hypermorphic mutation of phospholipase C, gamma2 acquired in ibrutinib-resistant CLL confers BTK independency upon Bcell receptor activation. Blood. 2015; 126:61-68. [PubMed: 25972157]

36. Song Z, Lu P, Furman RR, Leonard JP, Martin P, Tyrell L, et al. Activities of SYK and PLCgamma2 predict apoptotic response of CLL cells to SRC tyrosine kinase inhibitor dasatinib. Clin Cancer Res. 2010; 16:587-599. [PubMed: 20068106]

37. Haerzschel A, Catusse J, Hutterer E, Paunovic M, Zirlik K, Eibel H, et al. BCR and chemokine responses upon anti-IgM and anti-IgD stimulation in chronic lymphocytic leukaemia. Ann Hematol. 2016; 95:1979-1988. [PubMed: 27542958]

38. Yang C, Lu P, Lee FY, Chadburn A, Barrientos JC, Leonard JP, et al. Tyrosine kinase inhibition in diffuse large B-cell lymphoma: molecular basis for antitumor activity and drug resistance of dasatinib. Leukemia. 2008; 22:1755-1766. [PubMed: 18596745]

39. Lu P, Yang C, Guasparri I, Harrington W, Wang YL, Cesarman E. Early events of B-cell receptor signaling are not essential for the proliferation and viability of AIDS-related lymphoma. Leukemia. 2009; 23:807-810. [PubMed: 18987659]

40. Ma J, Xing W, Coffey G, Dresser K, Lu K, Guo A, et al. Cerdulatinib, a novel dual SYK/JAK kinase inhibitor, has broad anti-tumor activity in both ABC and GCB types of diffuse large B cell lymphoma. Oncotarget. 2015; 6:43881-43896. [PubMed: 26575169]

41. Cheng S, Coffey G, Zhang XH, Shaknovich R, Song Z, Lu P, et al. SYK inhibition and response prediction in diffuse large B-cell lymphoma. Blood. 2011; 118:6342-6352. [PubMed: 22025527] 
a

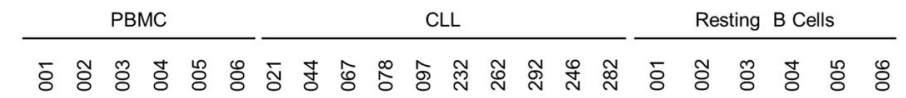

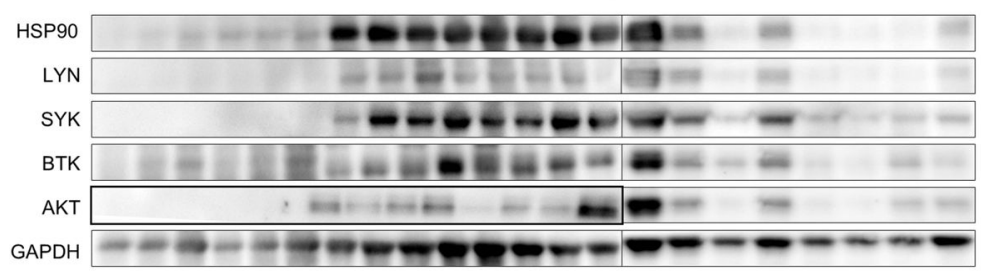

b
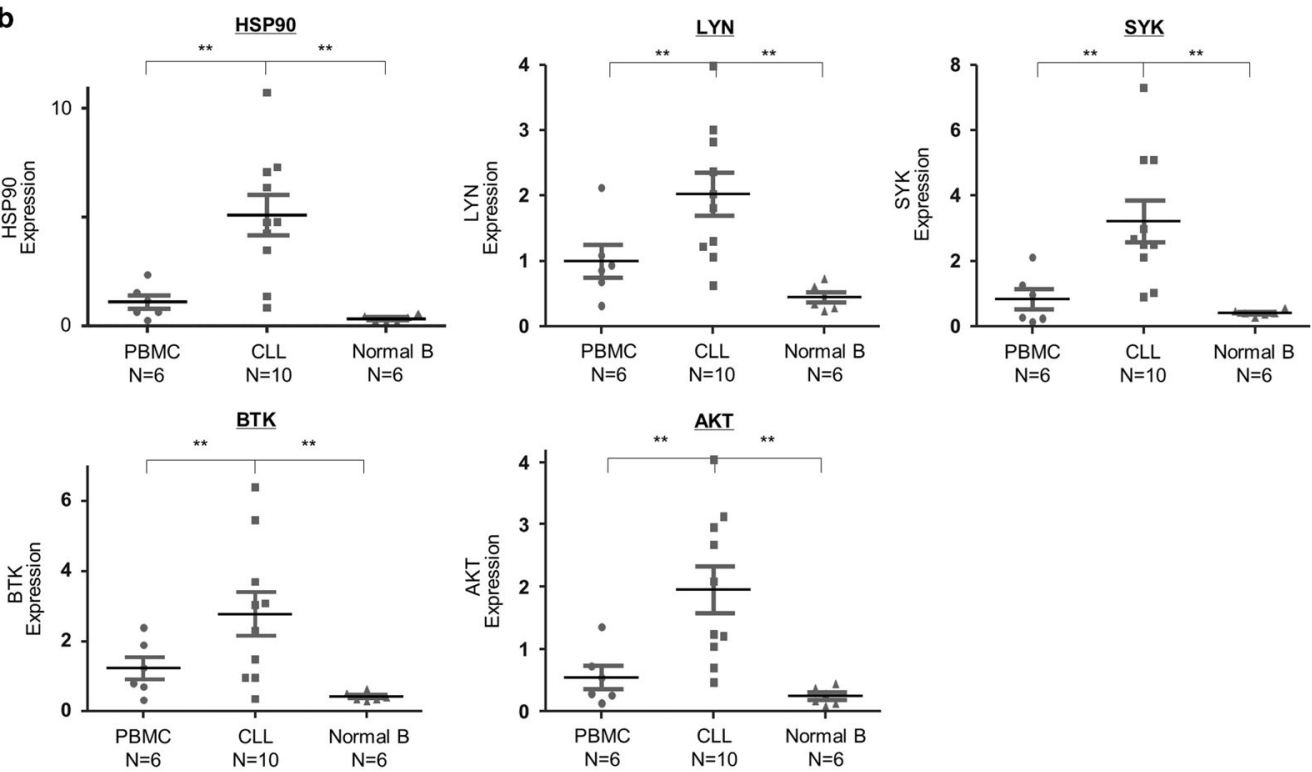

Figure 1.

HSP90 protein and BCR kinases are overexpressed in CLL compared with normal cell counterparts. (a) Western blot analysis of HSP90 and BCR kinases in CLL cells $(N=10)$, PBMCs $(N=6)$ and resting B cells $(N=6)$ from healthy donors. GAPDH, loading control. (b) Expression levels of HSP90 and BCR kinases were quantified and normalized to GAPDH. Bars represent means \pm s.e.m. $* * P<0.01$. 


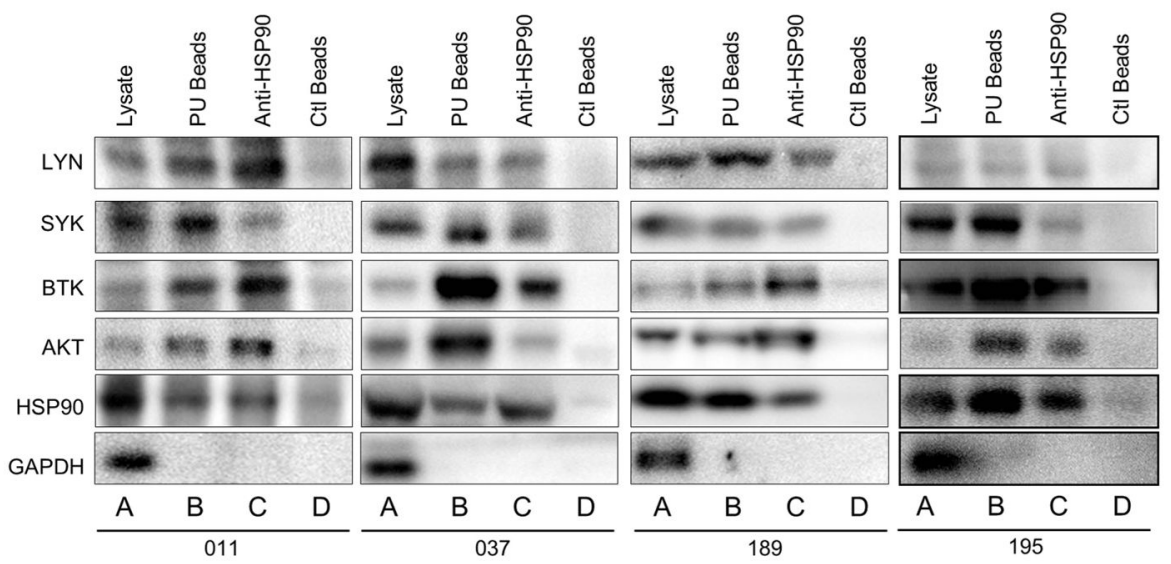

Figure 2.

BCR kinases are present in the multi-client HSP90 complex. Western blot of HSP90 and BCR kinases from cell lysates (lane As), chemical precipitates with PU-H71 beads (lane Bs), immunoprecipitates with anti-HSP90 beads (lane Cs) or control beads (lane Ds). 
a

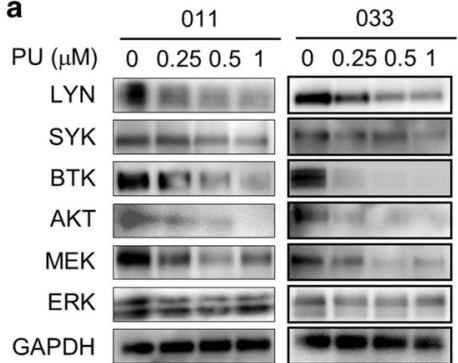

GAPDH -000

b

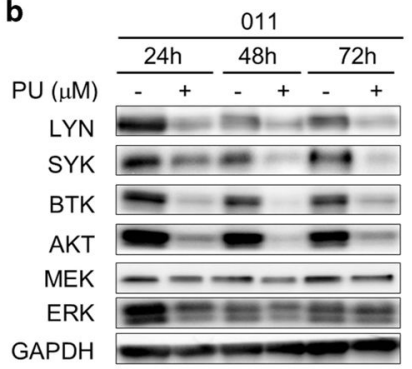

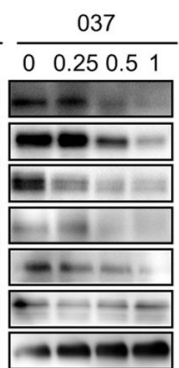
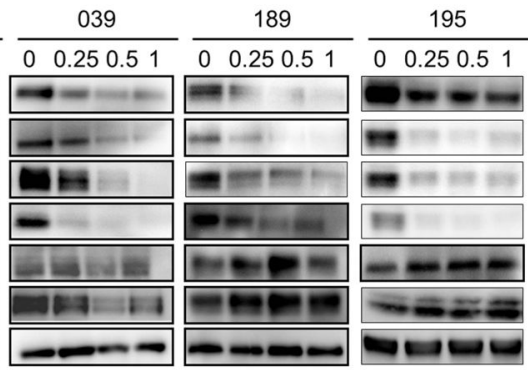

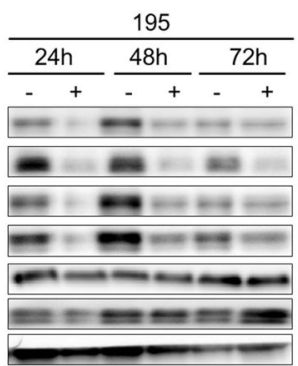

C
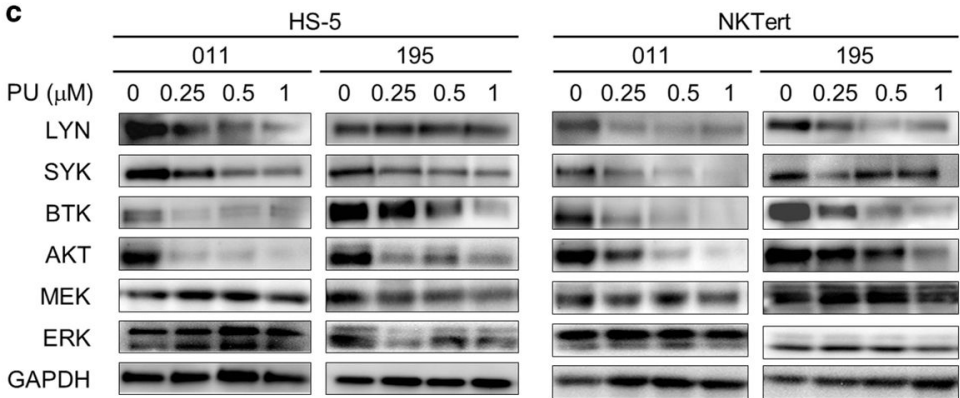

Figure 3.

Expression of BCR kinases are maintained by the chaperon function of HSP90. (a) Cells were treated with $0.5 \mu \mathrm{M}$ PU-H71 for the indicated hours. Protein lysates were probed for BCR signaling molecules. GAPDH, loading control. (b) Cells were treated with PU-H71 at indicated concentrations for $48 \mathrm{~h}$. (c) Cells co-cultured with HS-5 or NKTert were treated with PU-H71 at indicated concentrations for $48 \mathrm{~h}$. 
a

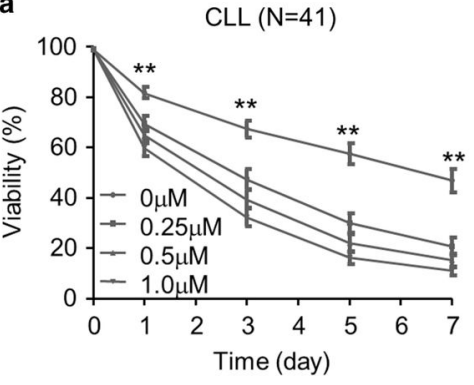

C

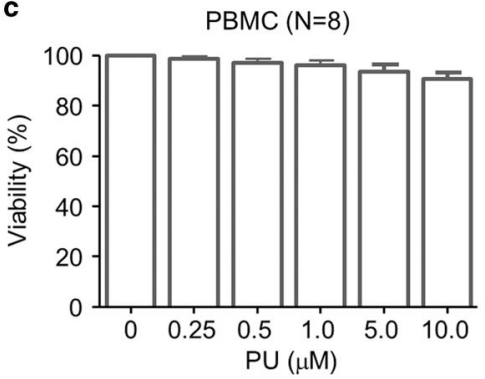

b

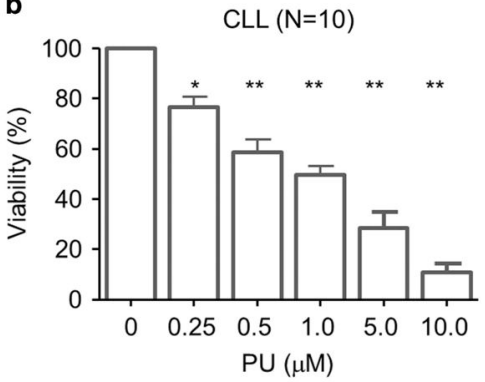

d

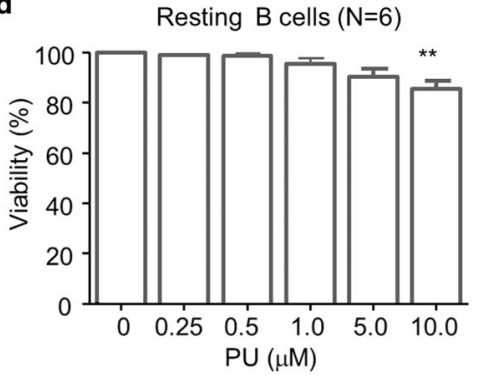

Figure 4.

PU-H71 significantly reduces the viability of CLL cells but has minimal effects on PBMC or resting B cells. (a) Time course of the viability of PU-H71-treated CLL cells $(N=44)$.

Percentage of PI-positive cells were analyzed by flow cytometry. (b) Dose dependence of the viability of PU-H71-treated CLL cells $(N=10)$. Cells were treated for $72 \mathrm{~h}$. (c) Effect of PU-H71 on PBMCs $(N=8)$. Cells were treated with the drug at indicated concentrations for $72 \mathrm{~h}$. (d) Effect of PU-H71 on resting B cells $(N=8)$. Cells were treated with the drug at indicated concentrations for 72 h. Bars represent means \pm s.e.m. $* P<0.05$; $* * P<0.01$. 
a

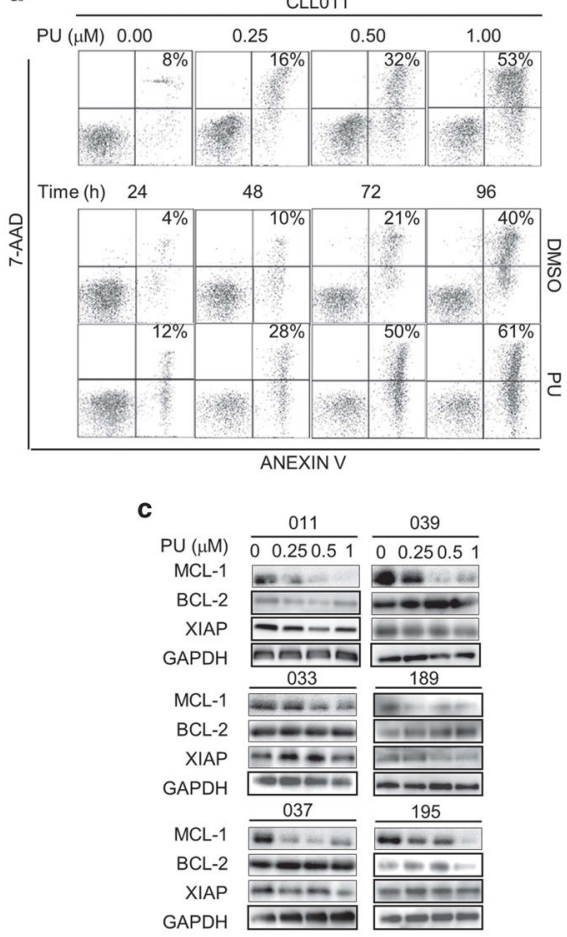

b

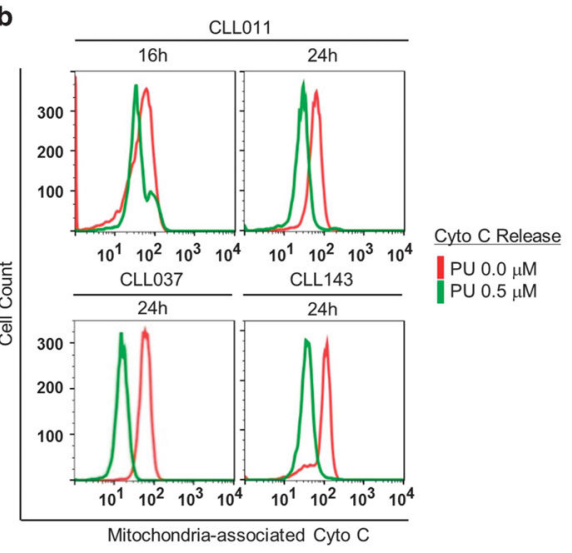

d

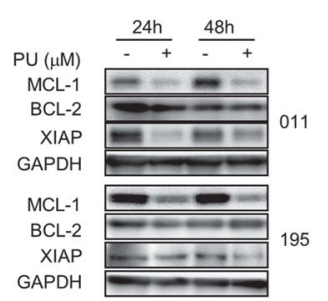

Figure 5.

PU-H71 reduces CLL survival through the induction of mitochondrial apoptosis. (a) CLL cells were treated with PU-H71 $(0-1 \mu \mathrm{M})$ for $48 \mathrm{~h}$ or $0.5 \mu \mathrm{M}$ for $24-96 \mathrm{~h}$. Apoptosis was measured by Annexin V/7-Aminoactinomycin D double staining. One of six cases was shown. (b) Cytochrome C release in PU-H71-treated CLL cells. Three of six cases are shown. Time and concentrations are indicated. (c) Western blot analysis of anti-apoptotic proteins in cells treated with PU-H71 at indicated concentrations for $48 \mathrm{~h}(N=6)$. (d) Western blot analysis of anti-apoptotic proteins in cells treated with $0.5 \mathrm{mM}$ of PU-H71 for 24 or $48 \mathrm{~h}$. 
a

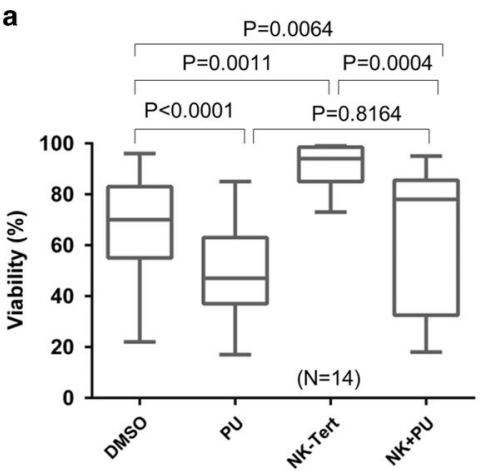

b

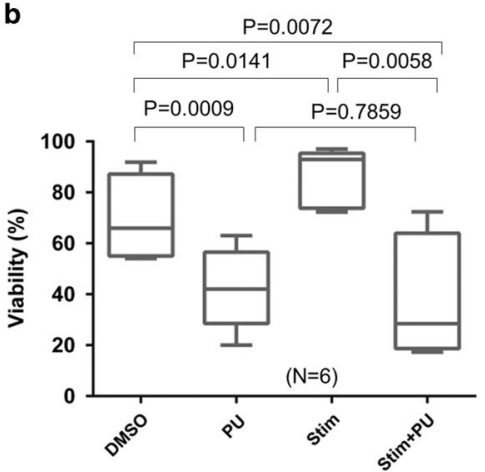

Figure 6.

PU-H71 antagonizes the survival signals from CLL microenvironment. (a) CLL cells with or without NKTert co-culture were treated with or without $0.5 \mu \mathrm{M}$ of PU-H71. (b) CLL cells with or without IL-4+CD40L were treated with or without $0.5 \mu \mathrm{M}$ of PU-H71. 
a

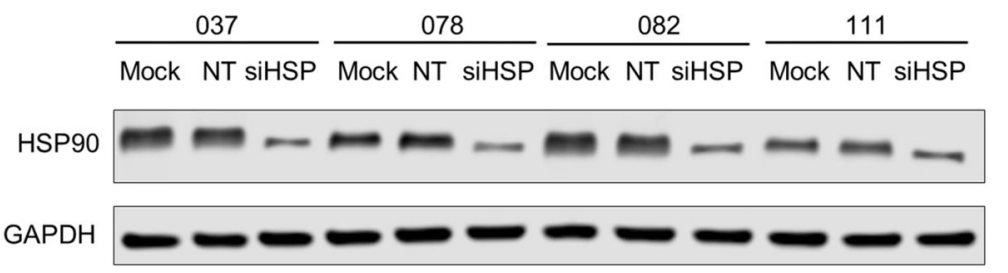

b

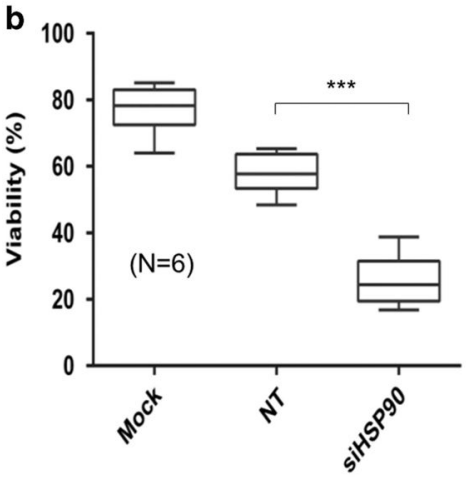

Figure 7.

HSP90 knockdown impairs CLL tumor cell viability. (a) Efficiency of HSP90 knockdown was assessed by western blot $(N=4)$. Mock, mock transfection; NT, cells were transfected with a pool of non-targeting siRNA. siHSP, cells were transfected with a pool of siRNA against HSP90. GAPDH, western loading control. (b) Effects of siRNA on CLL cell viability $(N=6)$. $* * * P<0.001$. 
a

d

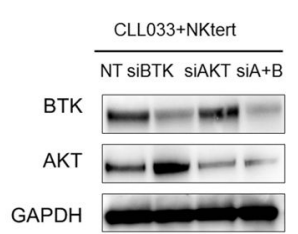

e

b

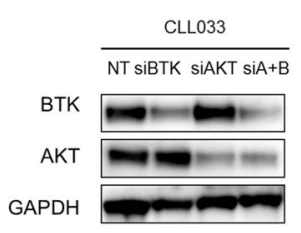

GAPDH
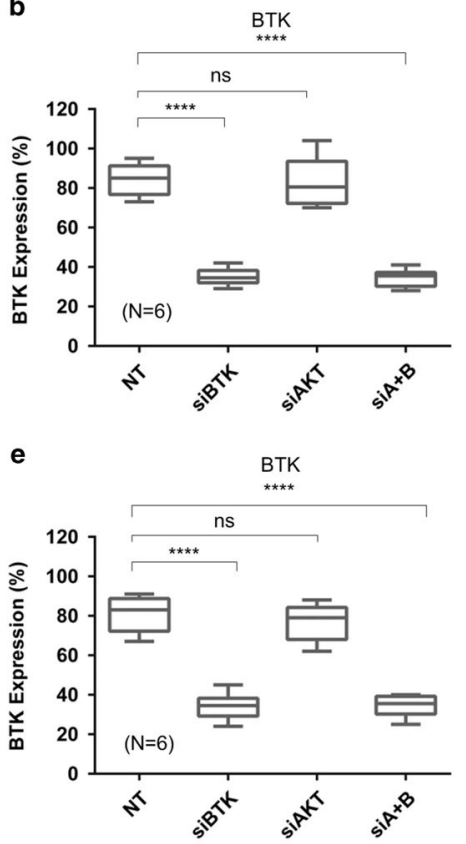

g

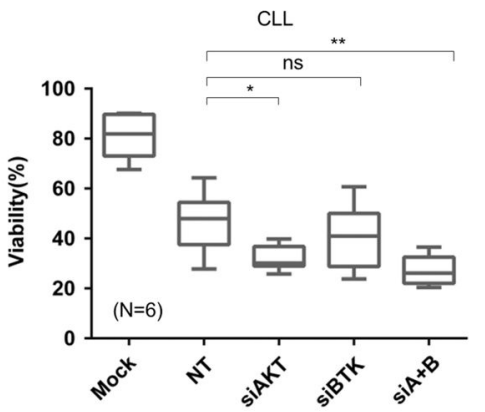

Figure 8. c

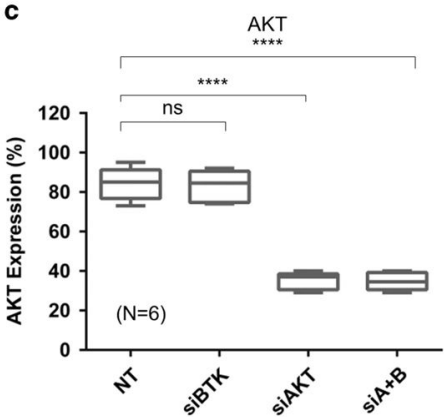

f

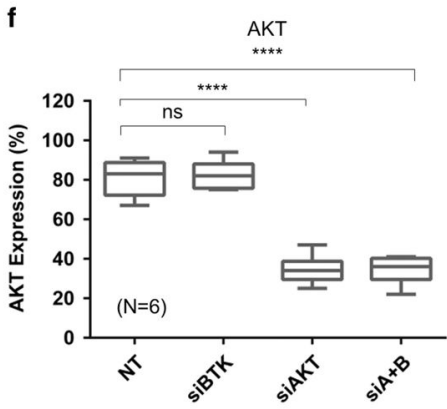

h

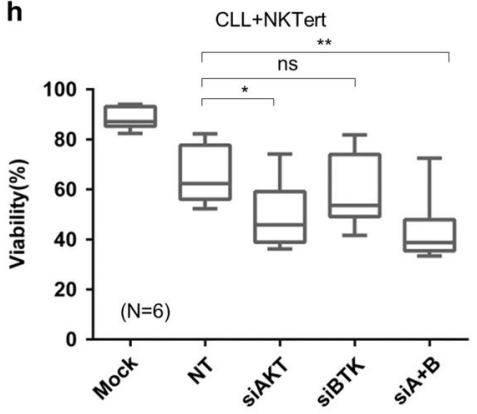

HSP90 client protein AKT, but not BTK, is essential for CLL viability. (a) CLL cells were transfected with siRNA against AKT, BTK, both or non-targeting siRNA (NT). Efficiency of siRNA knockdown was assessed by western blot. One of six representative case is shown. GAPDH, western loading control. (b, c) The abundance of the intended target, BTK, AKT or both was quantified by NIH ImageJ. (d) Efficiency of siRNA knockdown in the presence of NKTert co-culture. (e, f) Quantification of western blot shown in (d). (g, h) Effects of siRNA on CLL cell viability. (d) Effects of siRNA on CLL cell viability in the presence of NKTert co-culture $* P<0.05 ; * * P<0.01 ; * * * * P<0.0001$; ns, not significant. 